\title{
Do Different Methods Yield Equivalent Estimations of Brain Size in Birds?
}

\author{
Diego Ocampo a, b César Sánchez ${ }^{c} \quad$ Gilbert Barrantes $^{b}$ \\ ${ }^{a}$ Department of Biology, University of Miami, Coral Gables, FL, USA; ${ }^{\text {b }}$ Escuela de Biología, \\ Universidad de Costa Rica, San José, Costa Rica; ${ }^{C}$ Museum of Natural Science and Department of \\ Biological Sciences, Louisiana State University, Baton Rouge, LA, USA
}

\section{Keywords}

Fresh brain mass - Fixed brain mass - Endocranial volume ·

Body size $\cdot$ Hummingbirds

\begin{abstract}
The ratio of brain size to body size (relative brain size) is often used as a measure of relative investment in the brain in ecological and evolutionary studies on a wide range of animal groups. In birds, a variety of methods have been used to measure the brain size part of this ratio, including endocranial volume, fixed brain mass, and fresh brain mass. It is still unclear, however, whether these methods yield the same results. Using data obtained from fresh corpses and from published sources, this study shows that endocranial volume, mass of fixed brain tissue, and fresh mass provide equivalent estimations of brain size for 48 bird families, in 19 orders. We found, however, that the various methods yield significantly different brain size estimates for hummingbirds (Trochilidae). For hummingbirds, fixed brain mass tends to underestimate brain size due to reduced tissue density, whereas endocranial volume overestimates brain size because it includes a larger volume than that occupied by the brain.
\end{abstract}

(c) 2020 S. Karger AG, Basel
๑) 2020 S. Karger AG, Basel

\section{karger@karger.com}

www.karger.com/bbe

\section{Introduction}

The evolution of different behavioral patterns correlates with changes in brain size in a wide range of animals [West, 2014; Farris, 2015]. Consequently, relative brain size (the ratio of brain to body size) has extensively been used in species-specific and comparative studies that correlate behavioral capabilities with brain size across a wide range of taxonomic groups [Striedter, 2005; Eberhard and Wcislo, 2011]. In birds and mammals, the complexity of social systems, spatial navigation, and other behaviors seem to have influenced the evolution of their brain size [Nicolakakis and Lefebvre, 2000; Madden, 2001; Dukas, 2004; Lefebvre et al., 2004; Sol et al., 2005a; Møller, 2010; Shultz and Dunbar, 2010; Smaers et al., 2012; West, 2014; Lindsay et al., 2015]. However, some lines of evidence suggest that animals with small brains perform equally or perhaps more complex behaviors than animals with larger brains [Eberhard, 2007; Eberhard and Wcislo, 2011]. For instance, hummingbirds, the smallest-bodied birds have proven to be capable of song learning [Baptista and Schuchmann, 1990; Jarvis et al., 2000], a behavior that is thought to be neuronally demanding and which, in hummingbirds and other birds, is known to correlate
Diego Ocampo
Department of Biology
University of Miami
Coral Gables, FL 33146 (USA)
ocampov.diego@ gmail.com 
with the size of some specific regions of the brain (e.g., RA, HVC and LMAN) [Gahr, 2000]. Despite the fact that some behaviors are directly related to specific brain regions, relative brain size continues to be a common general predictor of behavioral complexity [Healy and Rowe 2007]. Unfortunately, it is still uncertain whether the different methods used over a wide range of studies to measure brain size are equivalent.

In most studies, brain size has been estimated using one of the following methods: (1) endocranial volume, (2) mass of brain tissue (after fixation of neural tissue with formaldehyde) [e.g., Iwaniuk and Arnold, 2004], (3) fresh brain mass [e.g., Garamszegi et al., 2002; Garamszegi and Eens, 2004; Møller et al., 2005], and (4) fitting an ellipsoid in the skull cavity [Madden, 2001]. Some investigations also combined brain size data estimated with different methods in order to increase the sample size and to address different ecological, behavioral, and evolutionary questions [e.g., Nealen and Ricklefs, 2001; Lefebvre et al., 2000, 2004; Sol et al., 2005b], without testing the equivalence of measurements obtained from different methods [Healy and Rowe, 2007]. This could be critical because some methods tend to overestimate brain size, while others seem to underestimate it.

Iwaniuk and Nelson [2002] showed, using regression analyses for 82 bird species, that endocranial volume and fixed brain mass yielded equivalent estimates of brain size, but equivalence between these methods and fresh brain mass has not yet been tested. The equivalence of all three methods deserves further research because results could differ between methods for different sets of species or families, particularly if they do not have the same range of variation in body size. Regressions calculated over a large number of species often mask some patterns, such as different slopes, intercepts, or dispersion of the residuals associated with particular families or species [Holloway, 2001].

Our study is designed to test whether three different methods, fresh brain mass, fixed brain mass, and endocranial volume, yield similar estimations of brain size. We also test whether statistical comparisons between methods for a data set yield similar results when applied to some groups of species. Results obtained from different methods could differ if they were used on a subset of species that extend over only a part of the range of brain and body sizes of a larger set of species. For instance, Iwaniuk and Nelson [2002] included some of the largest species of birds but did not include the smallest species. The relative error associated with the estimation of brain size tends to increase as brain size decreases; thus, estimating brain size of small species with statistical parameters (e.g., intercept and slope) derived from large species could result in imprecise measurements. We additionally conduct an independent analysis to test whether three different methods (fresh brain mass, fixed brain mass, and endocranial volume) yield equivalent estimations of brain size in hummingbirds (family Trochilidae), which includes the smallest birds, and explore whether the fixation process affects brain size estimates.

\section{Methods}

\section{Data Collection}

We compiled brain size estimates from 1,379 individuals in 164 bird species, from 48 families and 19 orders, allowing for 302 comparable estimations among three different methods (online suppl. Table S1; see www.karger.com/doi/10.1159/000509383 for all online suppl. material): endocranial volume (filling the skull with lead shot in all cases), fixed brain mass, and fresh brain mass. We use the term "fresh brain" for brains that were not chemically preserved, though they could have been frozen for $<7$ days before being weighed. Data on endocranial volume, fixed brain mass, and the corresponding body mass of these species were obtained from the literature (online suppl. Table S1). We excluded Bubo virginianus and Taenopygia guttata from Iwaniuk and Nelson [2002], because the body mass reported for these species, 177 and $5 \mathrm{~g}$, respectively, is far below the weight range reported elsewhere (B. virginianus $680-2,500 \mathrm{~g}$ [Holt et al., 2016]; T. guttata $\sim 10 \mathrm{~g}$ [Payne, 2016]). We transformed all endocranial volumes to mass (endocranial mass) by multiplying the volume by $1.036 \mathrm{~g} / \mathrm{mL}$. This value has been used in similar studies [e.g., Ebinger, 1995; Iwaniuk and Nelson, 2001], and is used here for comparative purposes.

Fresh brain and body masses of some species (online suppl. Table S1) were obtained from published data [Garamszegi et al., 2002] or from bird specimens collected between 2011 and 2015 from different regions of Costa Rica, as part of the collecting program of the Museo de Zoología, Universidad de Costa Rica (MZUCR). We stored collected birds wrapped in an air-tight, plastic bag at $-5{ }^{\circ} \mathrm{C}$ within the first $5 \mathrm{~h}$ after death and used only those adult specimens of both sexes that had been frozen for $<7$ days. We acknowledge that freezing birds, even for a short period, could dehydrate the tissue. However, we expect that wrapping birds in plastic bags reduces the freezing effect, and that the effect of freezing the birds would have a similar effect on body and brain. We determined total body mass to the nearest $0.1 \pm 0.05 \mathrm{~g}$ using a mechanical balance (Ohaus OH-750-S0) and total brain mass to the nearest $0.01 \pm 0.005 \mathrm{~g}$ using an American Scale ACPro-200. The brain of each specimen was carefully extracted from the skull by the same investigator (DO) and inspected for damage; only undamaged brains were used in the analyses. Brains included all the tissue enclosed in the braincase after removing the meninges, the optic nerve, and the spinal cord at the level of the foramen magnum. For the purpose of this study we use "size" and "mass" interchangeably as synonyms for brain size, and body size. 


\section{Comparisons between Methods}

We conducted pairwise comparisons between the three methods: endocranial mass, fixed brain mass, and fresh brain mass. Prior to comparisons, we divided the entire data set into subsets of species that had information for both brain and body size for at least two of the three methods: endocranial volume and fixed brain mass (90 spp.), endocranial volume and fresh brain mass (45 spp.), and fixed brain mass and fresh brain mass (19 spp.). We then tested whether brain mass, brain/body mass proportion, and body mass differ among methods, using paired $t$ tests. We used 6 phylogenetic generalized least-square (PGLS) models to estimate the log-log brain/body mass relationship in a phylogenetic controlled framework, in each of the data sets (e.g., a PGLS for the 90 species with data on endocranial volume). We tested differences between methods by extracting the slopes and intercepts from the PGLS models, and compared them using a $t$ test (e.g., interaction term and relative brain size among methods). We also used analysis of covariance (ANCOVA) to test for the effect of the method on the estimation of relative brain size; this test allows a comparison of the slopes and intercepts among methods in a single analysis but does not control for the phylogenetic relationships. Because the conclusions from the ANCOVA do not differ from those from the PGLS, here we only report the results from the phylogenetic controlled framework. In addition, we regressed brain mass versus body mass to represent graphically the effect of each method. For example, we used a PGLS to contrast brain/body mass proportion for endocranial mass (predictor variable) against brain/body mass proportion for fixed brain mass (response variable) for the same group of species. In these models, if both methods produce the same estimations of relative brain size, we expect a slope equal to 1 and an intercept equal to 0 .

For each of the PGLS models we used a consensus tree based on the majority rule of $50 \%$. We created the consensus tree using the package Phytools [Revell, 2012] and estimated the branch lengths using the method of least squares in the function "consensus.edges." Each consensus tree was based on 2000 phylogenetic trees obtained from birdtree.org [Jetz et al., 2012], using the Hackett backbone [Hackett et al., 2008]. All PGLS models were performed using the package Caper [Orme et al., 2012].

\section{Effect of Body Mass on Brain Size}

The body mass of the smallest bird in our data differs by nearly 4 orders of magnitude from the mass of the largest bird. Such a large range of variation allowed us to test the effect that the body size of bird species has on their brain sizes estimated by the endocranial mass and fixed brain mass methods. Based on body size, we split the data (90 species with data for both methods) into three groups with similar number of species. We averaged the body mass of each species and classified them into three groups, small birds: 6-55 g ( $n=29$ species), medium birds: $60-290 \mathrm{~g}$ ( $n=26$ species), and large birds: $310-35,800 \mathrm{~g}(n=35$ species $)$. To test the effect of the methods (interaction and intercept terms) on brain size estimation, we ran PGLS models, as described above, and tested for differences in slopes and intercepts using $t$ tests. The results did not differ from those obtained from ANCOVAs. We also evaluated the accuracy of model parameters (e.g., intercept and slope) obtained from the three groups. It is known that the dispersion of residuals around the predicted values affects the accuracy of the model in predicting the "true values." Thus, to test the effect of relative brain size on the residual dispersion, we ran a PGLS using the brain/body mass proportion from endocranial estimations against the fixed mass estimations and then compared residual variance with Levene's test among the three bird groups. We also tested whether the slopes and intercepts differed from the expected line (slope $=1$ and intercept $=0$ ) for each group of birds. The statistical parameters would be as expected if both methods (endocranial mass and fixed brain mass) yield the same estimations of brain size.

\section{Trochilidae Intrafamily Comparison}

The data available for hummingbirds allowed us to conduct comparisons between different groups of species with different methods used to estimate brain size. We conducted an analysis to examine the performance of these methods in estimating brain size on a family of birds that includes the smallest species. We first compared two groups of species with different estimations of brain and body mass: (1) brain mass estimated from endocranial volume and body size from skeletal measurements ( $n=20 \mathrm{spp}$. [Rehkämper et al., 1991]), and (2) brain mass estimated from endocranial volume and body size from weight of fresh specimens ( $n=15 \mathrm{spp}$., [Iwaniuk and Nelson, 2003]; Iwaniuk, pers. commun. [2018]). Specifically, we used an ANCOVA to compare the 2 sets of different species (online suppl. Table S1). Because these 2 sets varied greatly in their allometric relationships (slope and intercept), and the estimation of body mass based on skeletal traits used by Rehkämper et al. [1991] has not been validated for hummingbirds, we used only data from fresh specimens for further analyses.

We then used a set of hummingbird species (145 individuals from 47 species) that had data for body size and brain size estimated with at least one of these three methods - endocranial volume, fixed brain tissue, and fresh brain mass - to compare brain size estimations among methods. We first compared the body size (using ANOVA) of the species included in each of the three groups defined by the method of brain estimation. This analysis allowed us to test whether the species' body weights differ among the three groups. We then tested if the allometry (intercept and slope) of brain mass on body mass differed among the three groups (online suppl. Table S1): (1) 15 species with brain size estimated by endocranial volume [Iwaniuk and Nelson, 2003], (2) 9 species with brain size estimated with the fixed brain tissue method [Iwaniuk and Wylie, 2007], and (3) 24 species with fresh brain mass measurements. We first analyzed these data sets with an ANCOVA for the whole data set (54 brain-body records) to test for differences in slope and intercept among the three groups. We used similar statistical analyses to those used by Iwaniuk and Nelson [2002] for comparative purposes. Second, we ran a PGLS with the method of brain size estimation as a cofactor to control for phylogenetic relationship among 47 species. Because the PGLS does not allow for multiple values for the same species (e.g., brain size estimated with different methods for the same species), we randomly deleted one of the two estimates, resulting in a model based on 47 species, in which the brain size of each species was estimated using only one method.

\section{Fixation Effect}

We tested the effect of formaldehyde fixation by immersion of the brain mass of 12 brains of 11 species. Each fresh brain was carefully dissected from recently euthanized birds (never frozen) and the meninges removed. Each brain was weighed to the nearest $0.01 \mathrm{~g}$ (American Scale ACPro-200) and then maintained in a 10\% formaldehyde + phosphate buffer solution for 15 days. We tested 
Table 1. Results and parameters, PGLS models based on pairwise comparisons from different methods to estimate brain size and by body size

\begin{tabular}{|c|c|c|c|c|c|c|c|c|c|}
\hline Comparison & $F$ & $\mathrm{df}$ & $p$ value & $R^{2}$ & Lambda & Slope & SE & Intercept & SE \\
\hline \multicolumn{10}{|l|}{ Endocranial vs. fresh brain mass } \\
\hline Endocranial: body vs. brain & 638.4 & 1.43 & $<0.001^{*}$ & 0.94 & 1 & 0.66 & 0.03 & -1.09 & 0.08 \\
\hline Fresh: body vs. brain & 394.9 & 1.43 & $<0.001^{*}$ & 0.90 & 1 & 0.67 & 0.03 & -1.13 & 0.10 \\
\hline Brain mass: endocranial vs. fresh & 3,635 & 1.43 & $<0.001^{*}$ & 0.99 & 1 & 1.00 & 0.02 & -0.10 & 0.20 \\
\hline Brain/body mass: endocranial vs. fresh & 80.27 & 1.43 & $<0.001^{*}$ & 0.64 & 0 & 0.84 & 0.09 & 0.00 & 0.00 \\
\hline \multicolumn{10}{|l|}{ Fixed vs. fresh brain mass } \\
\hline Fixed: body vs. brain & 178.6 & 1.17 & $<0.001^{*}$ & 0.91 & 1 & 0.69 & 0.05 & -1.17 & 0.14 \\
\hline Fresh: body vs. brain & 193.4 & 1.17 & $<0.001^{*}$ & 0.91 & 1 & 0.71 & 0.05 & -1.23 & 0.14 \\
\hline Brain mass: fixed vs. fresh & 878.7 & 1.17 & $<0.001^{*}$ & 0.98 & 1 & 1.01 & 0.03 & -0.29 & 0.36 \\
\hline Brain/body mass: fixed vs. fresh & 55.87 & 1.17 & $<0.001^{*}$ & 0.75 & 0.7 & 0.84 & 0.11 & 0.00 & 0.00 \\
\hline \multicolumn{10}{|l|}{ Endocranial vs. fixed brain mass (total) } \\
\hline Endocranial: body vs. brain & 841.5 & 1.81 & $<0.001^{*}$ & 0.91 & 1 & 0.61 & 0.02 & -1.08 & 0.09 \\
\hline Fixed: body vs. brain & 606.4 & 1.81 & $<0.001^{*}$ & 0.88 & 0.83 & 0.65 & 0.03 & -1.20 & 0.10 \\
\hline Brain mass: endocranial vs. fixed & 4,162 & 1.81 & $<0.001^{*}$ & 0.98 & 0 & 0.95 & 0.01 & 0.28 & 0.11 \\
\hline Brain/body mass: endocranial vs. fixed & 381.9 & 1.81 & $<0.001^{*}$ & 0.82 & 0 & 0.90 & 0.05 & 0.00 & 0.00 \\
\hline \multicolumn{10}{|l|}{ Endocranial vs. fixed brain mass (small) } \\
\hline Endocranial: body vs. brain & 37.93 & 1.22 & $<0.001^{*}$ & 0.66 & 1 & 0.65 & 0.10 & -1.06 & 0.15 \\
\hline Fixed: body vs. brain & 35.69 & 1.22 & $<0.001^{*}$ & 0.60 & 1 & 0.76 & 0.13 & -1.26 & 0.19 \\
\hline Brain/body mass: endocranial vs. fixed & 12.78 & 1.22 & $0.002^{*}$ & 0.34 & 0.61 & 0.62 & 0.17 & 0.01 & 0.01 \\
\hline \multicolumn{10}{|l|}{ Endocranial vs. fixed brain mass (medium) } \\
\hline Endocranial: body vs. brain & 45.67 & 1.24 & $<0.001^{*}$ & 0.64 & 1 & 0.54 & 0.08 & -0.73 & 0.18 \\
\hline Fixed: body vs. brain & 33.18 & 1.24 & $<0.001^{*}$ & 0.56 & 0.87 & 0.54 & 0.09 & -0.74 & 0.21 \\
\hline Brain/body mass: endocranial vs. fixed & 57.98 & 1.24 & $<0.001^{*}$ & 0.70 & 0 & 0.95 & 0.12 & 0.00 & 0.00 \\
\hline \multicolumn{10}{|l|}{ Endocranial vs. fixed brain mass (large) } \\
\hline Endocranial: body vs. brain & 148.6 & 1.31 & $<0.001^{*}$ & 0.82 & 1 & 0.53 & 0.04 & -0.78 & 0.16 \\
\hline Fixed: body vs. brain & 63.46 & 1.31 & $<0.001^{*}$ & 0.66 & 0.69 & 0.52 & 0.06 & -0.75 & 0.22 \\
\hline Brain/body mass: endocranial vs. fixed & 159.7 & 1.31 & $<0.001^{*}$ & 0.83 & 0 & 1.01 & 0.08 & 0.00 & 0.00 \\
\hline \multicolumn{10}{|l|}{ Within Trochilidae } \\
\hline Endocranial: body vs. brain & 123 & 1.16 & $<0.001^{*}$ & 0.88 & 0.66 & 0.69 & 0.06 & -1.14 & 0.05 \\
\hline Fixed: body vs. brain & 16.56 & 1.7 & $0.005^{*}$ & 0.66 & 0 & 0.76 & 0.19 & -1.38 & 0.15 \\
\hline Fresh: body vs. brain & 387.6 & 1.18 & $<0.001^{*}$ & 0.95 & 0 & 0.67 & 0.03 & -1.21 & 0.02 \\
\hline
\end{tabular}

the effect of formalin fixation by comparing the weight of brains prior to immersion to their weight when patted dry 15 days later, right after the fixation period using a paired $t$ test. All statistical analyses were conducted in the statistical language R 3.0.2. (R Development Core Team 2019).

\section{Results}

\section{Comparison between Methods}

Endocranial Mass versus Fresh Brain Mass

The paired $t$ tests showed no difference in body size or brain size between the endocranial mass and fresh brain mass methods (body: $t_{44}=0.83, p=0.41$; brain: $t_{44}=-1.10$, $p=0.28)$, but the endocranial mass estimated a higher proportion of brain/body mass than that calculated with fresh brain mass $\left(t_{44}=-2.45, p=0.02\right)$. Brain size scaled similarly on body size for both methods (Table 1); neither intercepts nor slopes differed (intercepts: $t_{86}=0.35, p=$ 0.73 ; slopes: $t_{86}=0.16, p=0.88$ ). The slope from the relationship between brain mass calculated with both methods did not differ from the expected slope and intercept $\left(t_{43}=0.17, p=0.86\right.$ and $t_{43}=-0.50, p=0.62$ respectively; Table 1). In addition, the brain/body relationship from endocranial and fixed mass methods did not differ significantly from the expected slope $=0$ and intercept $=1$ $\left(t_{43}=1.75, p=0.09\right.$ and $t_{43}=0.70, p=0.49$; Table 1$)$; how- 
ever, this relationship showed that endocranial mass tends to produce larger estimates of brain size for small birds than the fresh brain mass method.

Fixed Brain Mass versus Fresh Brain Mass

We did not find significant differences (paired $t$ tests) in brain $\left(t_{18}=-1.57, p=0.13\right)$, body mass $\left(t_{18}=0.09, p=\right.$ $0.93)$, or brain/body proportion $\left(t_{18}=0.23, p=0.82\right)$ between methods. The relative brain size of 19 species estimated with fixed brain mass and fresh brain mass did not differ between these two methods (Table 1), neither slopes $\left(t_{34}=0.28, p=0.78\right)$ nor intercepts $\left(t_{34}=0.30, p=0.76\right)$. The slope and intercept estimated by the regression between fixed brain mass and fresh brain mass did not differ significantly from the expected allometric line of slope $=$ 1 and intercept $=0$ (slope: $t_{17}=0.21, p=0.84$; intercept: $t_{17}=-0.80, p=0.44$; Table 1 ) or for brain/body mass proportion (slope: $t_{17}=1.47, p=0.16$; intercept: $t_{17}=1.31$, $p=0.21 ;$ Table 1$)$.

\section{Endocranial Mass versus Fixed Brain Mass}

The body size, brain size, and proportion brain/body mass did not differ (paired $t$ tests) between the endocranial mass and fixed brain mass methods for 90 species $\left(t_{82}=1.19, p=0.24 ; t_{89}=-1.57, p=0.13 ; t_{82}=-0.49, p=\right.$ 0.62 , respectively). Relative brain size did not scale differently on body size between methods (Table 1); both methods yielded similar intercepts $\left(t_{162}=0.92, p=0.36\right)$ and slopes $\left(t_{162}=1.06, p=0.29\right)$. Nevertheless, the slope and intercept of the regression between endocranial mass and fixed brain mass methods differed from the expected parameters (slope $=1$, intercept $=0$ ) in both slope (slope: $\left.t_{81}=3.72, p<0.001\right)$ and intercept (intercept: $0.28 ; t_{81}=$ $2.61, p=0.01$ ). Similarly, the slope of brain/body mass proportion differed significantly from expectations (slope: $t_{81}=2.07, p=0.004$; intercept: $t_{81}=1.44, p=0.15$; Fig. 1). The regressions calculated suggested that the endocranial volume method estimates larger brains for small species than the fixed brain mass method.

\section{Effect of Body Mass on Brain Size}

When focusing on body mass categories, we found that the proportion of endocranial brain/body mass regressed against fixed brain/body mass proportion had a positive relationship in all cases. In addition, this regression showed a larger variation of residuals as the proportions of brain/body mass increase (Fig. 1). To test whether the dispersion of residuals from the PGLS differed along the curve, we divided the total sample into 3 body size categories and found that the greatest variance of re-

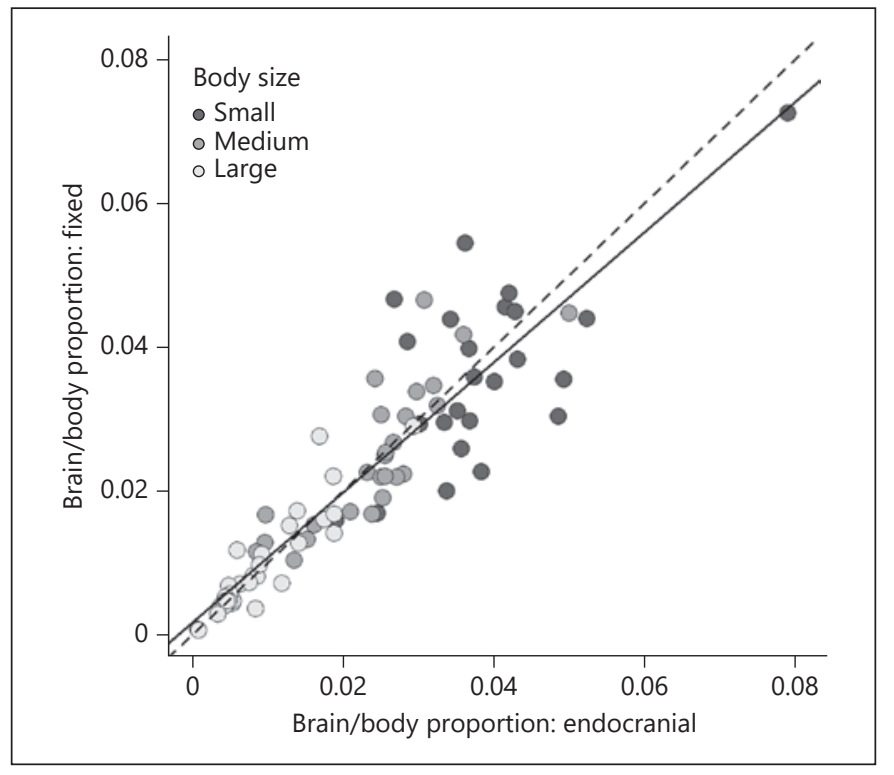

Fig. 1. Relationship between proportions of brain/body mass for fixed mass and endocranial brain mass for 90 species of birds (slope: $0.92, t=20.15, p<0.001$; intercept: $0.17, t=1.41, p=0.16$ ). Large birds (310-35,800 g), medium birds (60-290 g), and small birds (6-55 g). Dashed line shows the expected line (slope $=1$, intercept $=0)$.

siduals occurred in small birds (larger proportion brain/ body mass), whereas large birds presented the lowest variance of residuals (Levene's: $F_{2,80}=10.84, p<0.0001$ ).

Slopes and intercepts for relative brain size did not significantly differ between endocranial and fixed brain estimations for any of the three size groups (Fig. 2a-c; Table 1). The slopes in the log-log body versus brain size relationship did not differ between methods for all three groups (small: $t_{44}=0.65, p=0.52$; medium: $t_{48}=0.06, p=$ 0.95; large: $t_{62}=0.16, p=0.87$ ), nor did the intercepts (small: $t_{44}=0.81, p=0.42$; medium: $t_{48}=0.03, p=0.98$; large: $\left.t_{62}=0.12, p=0.90\right)$. The results of the analyses showed that the proportion of brain/body mass for fixed volume against endocranial brain mass (Table 1) for small birds had a lower slope but the same intercept than those expected (slope $=1$ and intercept $=0$; slope: $t=2.22, p=$ 0.04 , intercept: $t=1.35, p=0.19$; Fig. $2 \mathrm{~d}$ ). Medium-size birds did not differ from the expected slope and intercept (slope: $t=0.36, p=0.72$, intercept: $t=0.48, p=0.63$; Fig. 2e), nor did the large birds (slope: $t=1.44, p=0.89$, intercept: $t=0.18, p=0.86$; Fig. 2f).

\section{Trochilidae Intra-Family Comparison}

The two groups of hummingbird species with brain size estimated from endocranial volume, but with differ- 


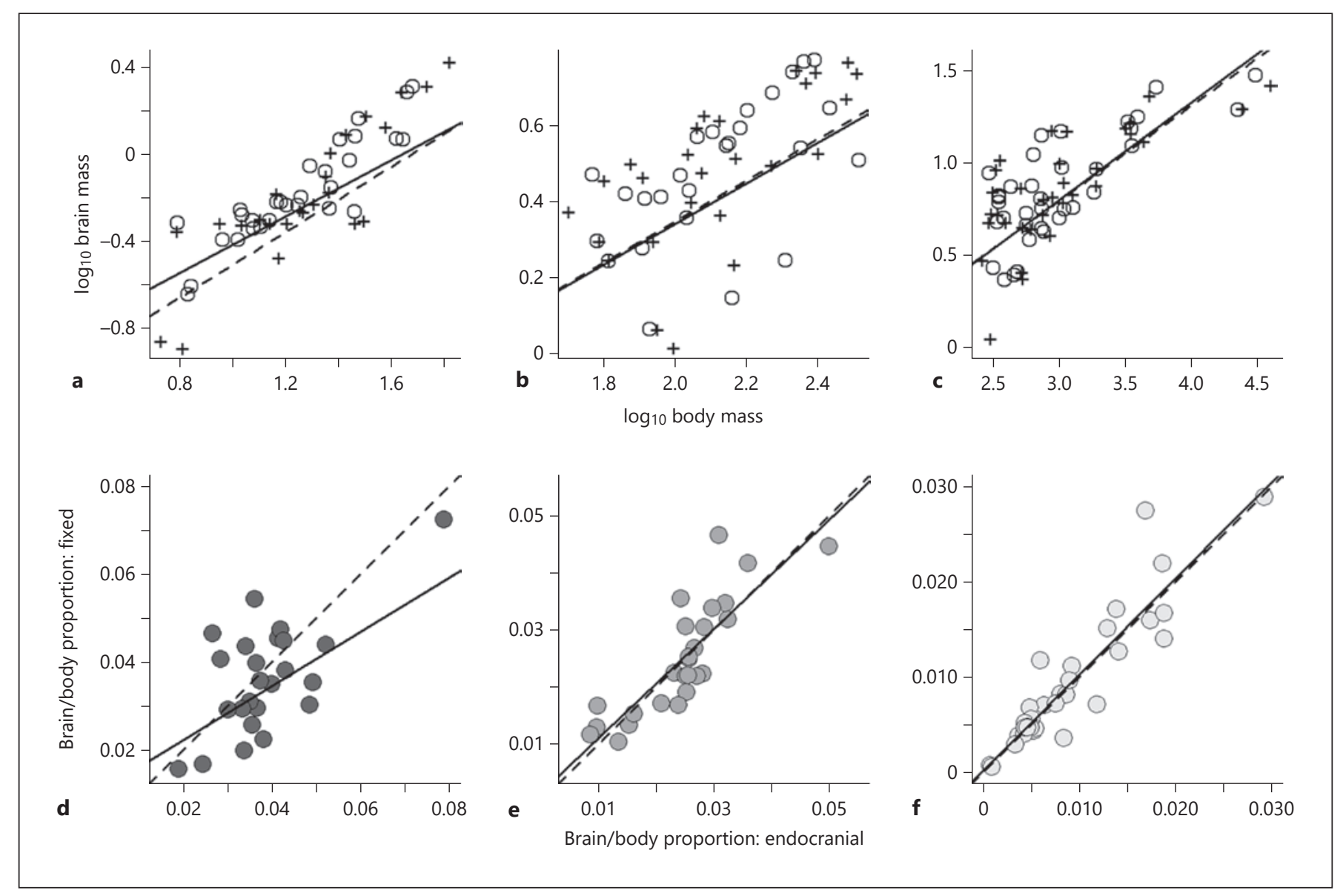

Fig. 2. The upper panel shows the log brain mass regressed against log body mass using data from two different methods of brain size estimation: endocranial mass (open circles, solid line) and fixed brain mass (plus sign, dotted line) for 29 small-sized birds (a), 26 medium-sized birds (b), and 35 large-sized birds (c). The lower panel shows the relationships between the proportion of brain/ body mass using data from two different methods of brain size estimation for the same groups of species: small-sized birds (d), medium-sized birds (e), and large-sized birds (f); the dashed lines show the expected lines $($ slope $=1$, intercept $=0$ ). ent methods to estimate body mass (from skeleton measurements or direct weight respectively), yielded different allometric relationships (ANCOVA: $F_{3,31}=50.90, p<$ $0.0001)$. Species whose body mass was estimated from skeleton measurements yielded a lower slope than those whose body mass was calculated from fresh specimens $\left(F_{1}=9.3, p=0.005\right)$, but intercepts were similar $\left(F_{1}=0.97\right.$, $p=0.33)$. Therefore, we used only the species with freshweighed body mass for further analyses.

There were no differences in body mass $\left(F_{2,51}=1.42\right.$, $p=0.25$ ) among the 3 sets of hummingbird species tested (22 species from endocranial volume, 9 species from fixed brain mass, and 24 species from fresh mass), or in brain mass $\left(F_{2,51}=1.93, p=0.15\right)$, but the brain/body proportions differed among the three groups $\left(F_{2,51}=17.32\right.$, $p<$ $0.0001)$. A Tukey HSD post hoc test showed differences among the three groups defined by the methods of brain size estimation $(q=2.41, p<0.05)$. Furthermore, the loglog relationship between body and brain size differed among the three groups (ANCOVA: $F_{5,48}=66.49, p<$ 0.0001 ; Table 1; Fig. 3$)$. There were no differences in the slopes among groups $\left(F_{2}=0.30, p=0.74\right.$; Fig. 3$)$, but they yielded different intercepts $\left(F_{2}=32.54, p<0.0001\right)$. Similarly, the PGLS showed significant differences among the intercepts, but not in the slopes estimated for the three methods $\left(F_{5,41}=56.56, p<0.0001, r^{2}=0.85\right)$. These results indicate that the endocranial mass method estimates larger brain sizes than the fixed brain mass method.

\section{Fixation Effect}

Brains immersed in formaldehyde drastically reduced their mass. After 15 days in formaldehyde fixation, the 


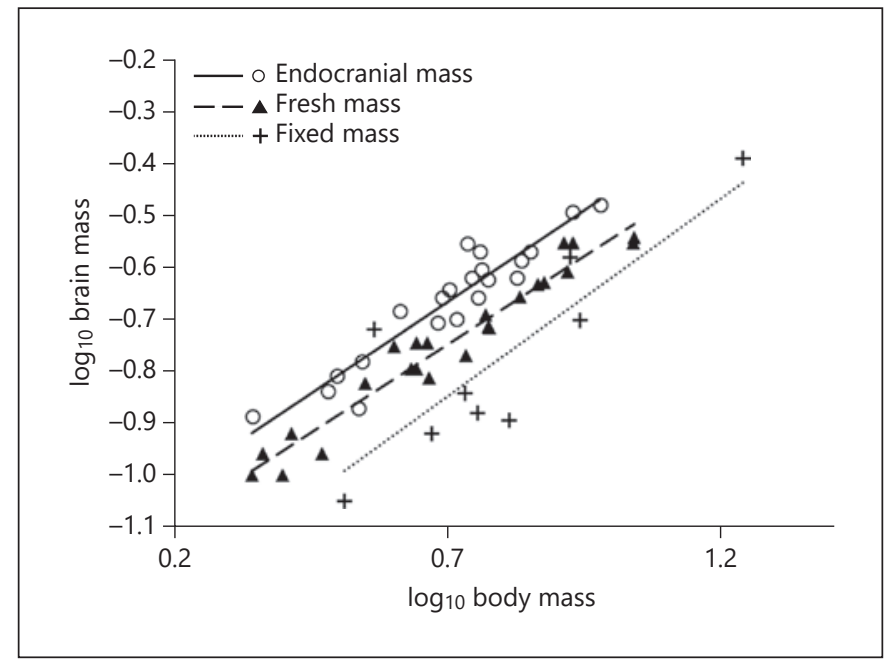

Fig. 3. log-log relationship of brain and body mass for 47 species of hummingbirds estimated with endocranial mass (open circles, solid line), fresh brain mass (filled triangles, dashed line), and fixed brain mass methods (plus sign, dotted line). Intercepts $\left(F_{2}=32.54, p<\right.$ $0.0001)$, but no slopes $\left(F_{2}=0.30, p=0.74\right)$, differ among methods.

mass of brains was significantly reduced $\left(t_{11}=-8.81, p<\right.$ 0.0001 ; Table 2 ). This change in weight by fixation would result in a lower elevation of the allometric line compared with fresh brain mass.

\section{Discussion}

In general, the three methods typically used to estimate brain size in birds and other vertebrates (endocranial mass, fixed brain mass, and fresh brain) yielded similar results. Pairwise comparisons between the three methods produced, in general, equivalent estimates of brain size. This accords with the results obtained by Iwaniuk and Nelson [2002] for endocranial mass and fixed brain mass estimated from formaldehyde-immersed brains. Our study also showed that the equivalence between endocranial mass and fixed brain mass methods was not a consequence of the broad range of body sizes measured: both methods still showed similar patterns after dividing species into three body size groups. However, estimates of brain size obtained with the endocranial mass method and the fixed brain mass method seems not be equivalent for small birds (lower slope and higher intercept than expected). The comparison between these two methods showed that the endocranial volume estimates larger proportions of brain/body size for small birds than the proportions obtained by the
Table 2. Differences in mass (g) of 12 brains after 15 days of fixation by immersion in formaldehyde $10 \%$

\begin{tabular}{llll}
\hline Species & $\begin{array}{l}\text { Fresh } \\
\text { mass }\end{array}$ & $\begin{array}{l}\text { Fixed } \\
\text { mass }\end{array}$ & Difference \\
\hline Geothlypis philadelphia & 0.52 & 0.41 & -0.11 \\
Ramphocelus costaricensis & 1.05 & 0.85 & -0.20 \\
Mionectes oleagineus & 0.49 & 0.40 & -0.09 \\
Coereba flaveola & 0.46 & 0.37 & -0.09 \\
Tyrannus melancholicus & 0.95 & 0.76 & -0.19 \\
Ramphocelus costaricensis & 1.03 & 0.82 & -0.21 \\
Myiarchus crinitus & 0.81 & 0.66 & -0.15 \\
Arremonops conirostris & 1.26 & 1.00 & -0.26 \\
Myiozetetes granadensis & 0.81 & 0.67 & -0.14 \\
Oreothlypis peregrina & 0.40 & 0.32 & -0.08 \\
Sporophila nigricollis & 0.51 & 0.41 & -0.10 \\
Xiphorhynchus susurrans & 1.17 & 0.97 & -0.20 \\
\hline
\end{tabular}

fixed brain mass method. Different factors other than the methods explored here, can affect the relative brain size estimated and the accuracy of these models. Additional variation could be attributed, for instance, to differences in size between sexes or seasonal variation in body mass [e.g., Scott et al., 1994]. To reduce variation caused by these factors, and thus, the residual dispersion in the allometric relationships, we averaged individual values for each species whenever possible. This procedure does not eliminate completely the variation due to individual differences, but it certainly reduces it. We are then confident that differences between curves, or differences in residual variation, are more likely due to the different methods used to estimate brain size.

The dispersion of residuals along the regression lines calculated for relative brain size of endocranial volume against fixed brain mass is greater in small than in large species. The dispersion or variation of the residuals around the regression line indicates the fit of the model to the data; thus, the smaller this variation the better the predictions of the model. Hence, in this particular case, the models indicate that the two methods estimate accurately the brain size for large birds, but accuracy decreases for small bird species. The greater dispersion of residuals in small species could be an effect of scale: the same measurement error would scale higher in small brains. For example, in a 2 -g bird with a 0.1 -g brain, the brain mass represents $5 \%$ of body mass, but if the measurement overestimates the brain mass by $0.05 \mathrm{~g}$, the brain mass would represent $7.5 \%$ of the body mass. However, in a 200 -g bird with a 4 -g brain, the brain proportion represents $2 \%$, and if the measurement was 


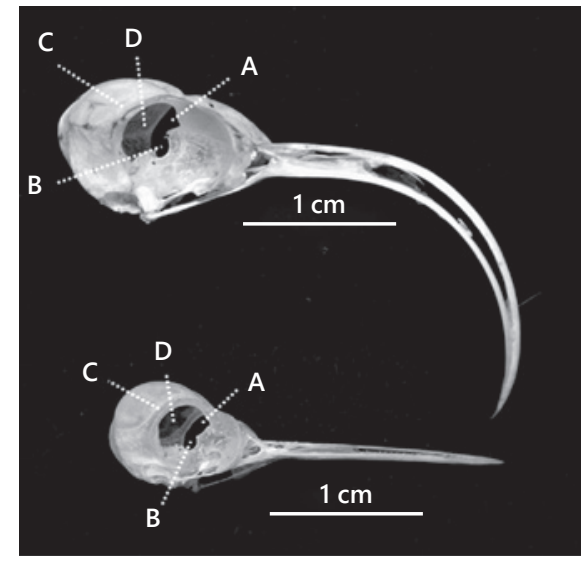

Fig. 4. Topography and nomenclature of the skull in the lateral view of 2 hummingbirds, Eutoxeres aquila without lower jaw (upper) and Selasphorus flammula, without lower jaw (lower). A, orbitocranial fonticulus; B, optic foramen; C, sulcus for cucullaris capitis muscle; D, braincase opened area.

equally overestimated by $0.05 \mathrm{~g}$, the resulted proportion brain/body mass would be $2.02 \%$.

We used data of the family Trochilidae because this family includes some of the smallest birds and falls out of the body size range of species analyzed by Iwaniuk and Nelson [2002]. The three groups of hummingbirds used in our analysis have the same range of body mass (2.2-17 g); thus, the differences in brain size among groups are not an effect of differences in body size. The endocranial volume method overestimates the mean (intercept) brain size. The brain size estimated by this method includes also the volume occupied by other tissue present in the braincase (e.g., brain ventricles) [Healy and Rowe, 2007]. To our knowledge, there are no published data on the volume of the ventricles in hummingbirds, which likely overestimates the brain size in this group of birds. Additionally, in hummingbirds the braincase does not ossify behind the eyes. The orbitocranial fonticulus is fused with the optic foramen [Zusi, 2013], so that the skull has an open space from the interorbital septum to the parietal bone, in the internal region of the sulcus for cucullaris capitis muscle (Fig. 4). This open area might affect the endocranial volume estimation by Rehkämper et al. [1991] and Iwaniuk and Nelson [2003].

Contrary to the endocranial volume method, the fixed brain mass underestimates brain size because the formaldehyde fixation process and $70 \%$ ethanol storage shrink the brain [Iwaniuk and Wylie, 2007]. In this study, formaldehyde fixation alone (no ethanol storage), by 15 days immersion, not only shrank the brains, but also reduced their density and so their mass, leading to an underestimation of brain mass.

The data on fresh brain mass from 24 hummingbird species showed low dispersion of the residuals, $R^{2}=0.95$. If we include data from the three methods, then the accuracy of the model decreases $\left(n=54, R^{2}=0.68\right)$. Thus, the accuracy of the model is more affected by the method used to estimate brain size than by the sample size included. Therefore, mixing data on brain size of hummingbirds estimated by different methods decreases precision of brain size estimation across species.

We focused on brain mass, because it is one of the easiest and most common measurements for brain size, but estimations of volume might be equally affected. We conclude that, in general, brain size data from endocranial volume, fixed, and fresh brain mass methods render equivalent estimations of brain mass for birds. However, among hummingbirds, the estimation of brain size was affected by the method used to obtain these estimates. The fixation by immersion method underestimated brain size because it reduced tissue density, while the endocranial volume method overestimated brain size since this estimation included sections of the braincase that were not occupied by brain tissue. We used data from different sources and acknowledge that factors other than the method, such as sex, mating season, subspecies, and individual variation, may affect the estimation of brain size. However, those factors are likely similarly distributed among the data obtained for the three methods used to estimate brain size, reducing the bias of data obtained by a particular method.

Combining data on brain size obtained with different methods would at least increase residual dispersion and so decrease predicting accuracy of the model, particularly in hummingbirds, because in these birds the pattern of skull ossification makes it difficult to have an accurate estimation of brain volume. We consider that combining data obtained from different methods of brain size estimation could still be useful to answer general questions or to explore general patterns, but combining data to answer more specific questions or describe species-specific behavioral patterns (e.g., courtship behavior within a closely related group of species) could affect the results since small variation in brain size estimation could modify the conclusions of the study. For hummingbirds, the fresh brain mass method renders the most accurate estimation of brain size. 


\section{Acknowledgment}

We thank W. Eberhard for assistance in the design of the study; A.N. Iwaniuk for clarifications on the methods used; J.L. Cantalapiedra, D. Warren, and C. Searcy for comments on the statistical methods; J.A. Uy and W.A. Searcy lab members, from the University of Miami, for comments that improved a previous version of the manuscript; J.V. Remsen and W.A. Searcy for help with editing the paper; and reviewers for helpful comments and suggestions.

\section{Statement of Ethics}

This research was conducted under SINAC permits: 1812010-SINAC; 001-2012-SINAC; 007-2013-SINAC, and SINACSE-GASP-PI-R-O72-2014; and following the AOS Guidelines to the Use of Wild Birds in Research (www.nmnh.si.edu/BIRDNET/ guide).

\section{Conflict of Interest Statement}

The authors have no conflicts of interest to declare.

\section{Funding Sources}

This research was mainly funded by the authors and partially funded be AMNH Frank Chapman Memorial Fund (to C.S.) and the LSUMNS Big Day Fund (to C.S.). None of the funders had any influence on the content or required approval of the final manuscript.

\section{Author Contributions}

D.O. and G.B. formulated the questions; D.O. and C.S. collected the data; D.O. analyzed the data and wrote the paper; C.S. and G.B. substantially edited the paper.

\section{References}

Baptista LF, Schuchmann K. Song learning in the Anna hummingbird (Calypte anna). Ethology. 1990;84(1):15-26.

Dukas R. Evolutionary biology of animal cognition. Annu Rev Ecol Evol Syst. 2004;35(1): $347-74$.

Eberhard WG. Miniaturized orb-weaving spiders: behavioural precision is not limited by small size. Proc Biol Sci. 2007 Sep;274(1622): 2203-9.

Eberhard WG, Wcislo WT. Grade changes in brain-body allometry: Morphological and behavioural correlates of brain size in miniature spiders, insects and other invertebrates. In: Casas J, editor. Adv Insect Physiol 40. pp. 155-214.

Ebinger P. Domestication and plasticity of brain organization in mallards (Anas platyrhynchos). Brain Behav Evol. 1995;45(5):286-300.

Farris SM. Evolution of brain elaboration. Philos Trans R Soc Lond B Biol Sci. 2015 Dec: 370(1684):20150054

Gahr M. Neural song control system of hummingbirds: comparison to swifts, vocal learning (Songbirds) and nonlearning (Suboscines) passerines, and vocal learning (Budgerigars) and nonlearning (Dove, owl, gull, quail, chicken) nonpasserines. J Comp Neurol. 2000 Oct;426(2):182-96.

Garamszegi LZ, Eens M. Brain space for a learned task: strong intraspecific evidence for neural correlates of singing behavior in songbirds. Brain Res Brain Res Rev. 2004 Mar;44(2-3) $187-93$.

Garamszegi LZ, Møller AP, Erritzøe J. Coevolving avian eye size and brain size in relation to prey capture and nocturnality. Proc Biol Sci. 2002 May;269(1494):961-7.

Hackett SJ, Kimball RT, Reddy S, Bowie RC, Braun EL, Braun MJ, et al. A phylogenomic study of birds reveals their evolutionary history. Science. 2008 Jun;320(5884):1763-8.
Healy SD, Rowe C. A critique of comparative studies of brain size. Proc Biol Sci. 2007 Feb; 274(1609):453-64.

Holloway RL. Does allometry mask important brain structure residuals relevant to speciesspecific behavioral evolution? Behav Brain Sci. 2001;24(2):286-7.

Holt DW, Berkley R, Deppe C, Enríquez-Rocha P, Petersen JL, Rangel-Salazar JL, et al. Great Horned Owl (Bubo virginianus). In: del Hoyo J, Elliott A, Sargatal J, Christie DA, de Juana E, editors. Handbook of the Birds of the World Alive. Barcelona: Lynx Edicions; 2016. Available from: http://www.hbw.com/ node/55006.

Iwaniuk AN, Arnold KE. Is cooperative breeding associated with bigger brains? A comparative test in the Corvidae (Passeriformes). Ethology. 2004;110(3):203-20.

Iwaniuk AN, Nelson JE. A comparative analysis of relative brain size in waterfowl (Anseriformes). Brain Behav Evol. 2001 Feb;57(2): 87-97.

Iwaniuk AN, Nelson JE. Can endocranial volume be used as an estimate of brain size in birds? Can J Zool. 2002;80(1):16-23.

Iwaniuk AN, Nelson JE. 2003. Developmental differences are correlated with relative brain size in birds: a comparative analysis. Can J Zool. 2003;81:1913-1928.

Iwaniuk AN, Wylie DR. Neural specialization for hovering in hummingbirds: hypertrophy of the pretectal nucleus Lentiformis mesencephali. J Comp Neurol. 2007 Jan;500(2):211-21.

Jarvis ED, Ribeiro S, da Silva ML, Ventura D, Vielliard J, Mello CV. Behaviourally driven gene expression reveals song nuclei in hummingbird brain. Nature. 2000 Aug;406(6796): 628-32.

Jetz W, Thomas GH, Joy JB, Hartmann K, Mooers AO. The global diversity of birds in space and time. Nature. 2012;491:444-448.
Lefebvre L, Nicolakakis N, Boire D. Tools and brains in birds. Behaviour. 2002;139:939-73.

Lefebvre L, Reader SM, Sol D. Brains, innovations and evolution in birds and primates. Brain Behav Evol. 2004;63(4):233-46.

Lindsay WR, Houck JT, Giuliano CE, Day LB. Acrobatic courtship display coevolves with brain size in manakins (Pipridae). Brain Behav Evol. 2015;85(1):29-36.

Madden J. Sex, bowers and brains. Proc Biol Sci. 2001 Apr;268(1469):833-8.

Møller AP. Brain size, head size and behaviour of a passerine bird. J Evol Biol. 2010 Mar;23(3): 625-35.

Møller AP, Erritzøe J, Garamszegi LZ. Covariation between brain size and immunity in birds: implications for brain size evolution. J Evol Biol. 2005 Jan;18(1):223-37.

Nealen PM, Ricklefs RE. Early diversification of the avian brain: body relationship. J Zool (Lond). 2001;253(3):391-404.

Nicolakakis N, Lefebvre L. Forebrain size and innovation rate in European birds: Feeding, nesting and confounding variables. Behaviour. 2000;137(11):1415-29.

Orme D, Freckleton RP, Petzoldt T, Fritz S, Isaac $\mathrm{N}$, Pearse W. Comparative analyses of phylogenetics and evolution in $\mathrm{R}$. $\mathrm{R}$ version 0.5.2. cran.r-project.org/web/packages/caper/index. 2012.

Payne R. Timor Zebra Finch (Taeniopygia guttata). In: del Hoyo J, Elliott A, Sargatal J, Christie DA, de Juana E, editors. Handbook of the Birds of the World Alive. Barcelona: Lynx Edicions; 2016. Available from: http:// www.hbw.com/node/61172.

Rehkämper G, Schuchmann KL, Schleicher A, Zilles K. Encephalization in hummingbirds (Trochilidae). Brain Behav Evol. 1991;37(2): 85-91. 
Revell LJ. phytools: an R package for phylogenetic comparative biology (and other things). Methods Ecol Evol. 2012;3(2):217-23.

Schultz S, Dunbar RI. Social bonds in birds are associated with brain size and contingent on the correlated evolution of life-history and increased parental investment. Biol J Linn Soc Lond. 2010;100(1):111-23.

Scott I, Mitchell PI, Evans PR. Seasonal changes in body mass, body composition and food requirements in wild migratory birds. Proc Nutr Soc. 1994 Nov;53(3):521-31.
Smaers JB, Dechmann DK, Goswami A, Soligo C, Safi K. Comparative analyses of evolutionary rates reveal different pathways to encephalization in bats, carnivorans, and primates. Proc Natl Acad Sci USA. 2012 Oct;109(44): 18006-11.

Sol D, Duncan RP, Blackburn TM, Cassey P, Lefebvre L. Big brains, enhanced cognition, and response of birds to novel environments. Proc Natl Acad Sci USA. 2005a Apr;102(15):54605
Sol D, Lefebvre L, Rodríguez-Teijeiro JD. Brain size, innovative propensity and migratory behaviour in temperate Palaearctic birds. Proc Biol Sci. 2005b Jul;272(1571):1433-41.

Striedter GF. Principles of brain evolution. Sunderland (MA): Sinauer Associates Inc.; 2005. p. 436.

West HJ. The evolution of large brain size in birds is related to social, not genetic, monogamy. Biol J Linn Soc Lond. 2014;111(3):668-78.

Zusi R. Introduction to the skeleton of hummingbirds (Aves: Apodiformes, Trochilidae) in functional and phylogenetic contexts. Ornithol Monogr. 2013;77(1):1-94. 